Revista Brasileira de Agricultura Irrigada v.12, nº6, p. 3069 - 3077, 2018

ISSN 1982-7679 (On-line)

Fortaleza, CE, INOVAGRI - http://www.inovagri.org.br

DOI: $10.7127 /$ rbai.v12n6001028

Protocolo 1028.18 - 10/10/2018 Aprovado em 13/01/2019

\title{
ESTABILIDADE TEMPORAL DA VARIABILIDADE ESPACIAL DA UMIDADE DO SOLO OBTIDA NO CAMPO E NO LABORATÓRIO
}

\author{
Anderson Takashi Hara ${ }^{1}$, Antônio Carlos Andrade Gonçalves ${ }^{2}$
}

\begin{abstract}
RESUMO
Em projetos de irrigação, uma curva de retenção de água no solo que represente a condição média do domínio experimental é importante para garantir a exatidão da lâmina de irrigação a ser aplicada na cultura. Contudo, a coleta aleatória de amostras indeformadas do solo pode não possibilitar a inferência de uma curva média. Dois experimentos foram realizados, avaliando a distribuição de valores da variável umidade do solo em diferentes posições em uma malha de amostragem, em vários momentos ao longo de um processo de secamento do solo. Em cada posição foi coletado amostras indeformadas para determinação da curva de retenção de água no solo. Análises de estabilidade temporal e geoestatística foram realizadas. As variáveis analisadas apresentaram continuidade espacial sendo também correlacionadas espacialmente. A análise de estabilidade temporal comprovou que a umidade do solo obtido no campo e originada da curva de retenção apresentam estabilidade temporal e o estudo da umidade obtida no campo pode ser utilizada para inferir a posição amostral que represente a curva de retenção média de um domínio.
\end{abstract}

Palavras-chave: curva de retenção, geoestatística, semivariograma, semivariograma cruzado.

\section{TEMPORAL STABILITY OF SPATIAL VARIABILITY OF SOIL MOISTURE OBTAINED IN THE FIELD AND IN THE LABORATORY}

\begin{abstract}
In irrigation projects, a soil water retention curve that represents the average condition of the experimental domain is important to ensure the accuracy of the irrigation sheet to be applied in the crop. However, a random sample of undisturbed soil samples can not allow the inference of an average. Two experiments were carried out, evaluating a distribution of variable samples of the soil samples in the mesh samples, in many moments to a long process of drying the soil. In each position the samples were collected to determine the water retention curve in the soil. Temporal stability and geoestatistics analyzes were performed. that the soil moisture obtained

\footnotetext{
1 Engenheiro Agrônomo, Doutor, UEM, Maringá-PR, Brasil. e-mail: haratakashi1987@gmail.com

2 Engenheiro Agrícola, Prof. Doutor, Departamento de agronomia, UEM, Maringá-PR, Brasil, Av. Colombo, N ${ }^{\circ}$ 5790 - Bloco J35, e-mail: aca.goncalves@ gmail.com
} 
in the field and originated from the retention curve presents temporal stability and the study of the moisture obtained in the field can be used to infer the sample position that represents the mean retention curve of a domain.

Keywords: cross-semivariogram, geoestatistics, retention curve, semivariogram.

\section{INTRODUÇÃO}

O conhecimento da condição média de um domínio da umidade do solo no campo $(\theta)$ e a curva de retenção são imprescindíveis para um adequado manejo de um sistema de irrigação. Essa condição tendo sido corriqueiramente estimada por meio de uma amostragem aleatória de alguns pontos no domínio. No entanto essa estratégia pode gerar resultados insatisfatórios uma vez que $\theta$ (GONÇALVES et al., 1999; REZENDE et al., 2002; GONÇALVES et al., 2010; GUIMARÃES et al., 2010; SALVADOR et al., 2012) e a umidade do solo obtida da curva de retenção de água no solo ( $\theta$ CRAS) (MOHANTY; MOUSLI, 2000) pode apresentar variabilidade espacial. Além disso, para que haja uma qualidade na inferência, deve-se adotar grande números de pontos amostrados. Uma vez que amostragem em diferentes pontos se necessita de considerável tempo e recursos, tornase conveniente que por uma única posição proporcionasse o conhecimento do valor médio da variável a ser medida, admitindo uma certa margem de erro associado. Vachaud et al., (1985) procurando superar as dificuldades do impasse descrito anteriormente, desenvolveu a metodologia da estabilidade temporal, que visa determinar a posição no espaço adequado para representar uma condição média dentro de um domínio.

O objetivo do trabalho é testar as seguintes hipóteses: A $\theta$ e $\theta$ CRAS apresentam estabilidade temporal; uma determinada posição que possibilita a predição da condição média da $\theta$ no campo de um dado domínio pode ser adotada na obtenção da CRAS média.

\section{MATERIAL E MÉTODOS}

O presente trabalho foi realizado em duas áreas experimentais (A e B) em diferentes classes de solo, conduzidos por Gonçalves et al., (1999) e Guimarães et al., (2010) respectivamente. A área experimental (A) foi compreendida por um quadrante de uma área irrigada por um sistema pivô-central, com declividade média de aproximadamente $2 \%$ na sua direção bissetriz. $\mathrm{O}$ solo da área foi classificado como Argissolo vermelho apresentando os seguintes teores de areia, silte e argila: 337; 263; $404 \mathrm{~g} \mathrm{~kg}^{-1}$, respectivamente. A superfície do solo foi submetida a uma gradagem e posteriormente foi demarcada uma transeção, segundo um raio da área, com 141,42 metros, em solo nu, na direção da menor declividade do terreno. Os pontos foram marcados a cada $2,83 \mathrm{~m}$. $\mathrm{Na}$ transeção foram instaladas 50 sondas de nêutrons monitorando a umidade do solo em base volume $(\theta)$ na profundidade de $0,15 \mathrm{~m}$ ao longo de um processo de secagem do solo, sendo que o valor de $\theta$ inicial estava na capacidade de campo. Posteriormente a esta etapa experimental, foi procedido a coleta de amostras indeformadas, ao lado de cada posição em que situavam as sondas de Nêutrons, com anéis volumétricos de $100 \mathrm{~cm}^{3}$ do solo a $0,20 \mathrm{~m}$ de profundidade, e no laboratório, foram submetidas as amostras nas tensões $0,10,20$, 40, 100, 300, 800, 4000 e $15000 \mathrm{hPa}$, obtendo-se o valor da umidade em base volume ( $\theta$ CRAS) das 50 amostras.

A área experimental B apresentava uma área de 15 x $48 \mathrm{~m}$, com sistema de irrigação por aspersão. O solo da área é classificado como Nitossolo Vermelho distroférrico apresentando os seguintes teores de areia, silte e argila: 175; 90; 735 $\mathrm{g} \mathrm{kg}^{-1}$, respectivamente. A área experimental foi dividida em 80 quadrículas de dimensão de 3 x 3 $\mathrm{m}$. No centro de cada quadrícula foi instalado verticalmente uma sonda de TDR com $0,20 \mathrm{~m}$ de comprimento para o monitoramento do $\theta$. Após 50 dias da semeadura da cultura do feijoeiro, foi monitorado a $\theta$ por 12 dias ao longo do processo de secagem do solo, considerando o valor de $\theta$ inicial próximo à capacidade de campo. Após a colheita, realizada no dia 10 de agosto de 2006, procedeu-se a amostragem do solo no centro de cada parcela, na porção intermediária da camada de 0-0,20 m, utilizando anéis volumétricos de 100 $\mathrm{cm}^{3}$, totalizando 80 amostras de solo indeformadas. 
No laboratório foram submetidas as tensões 010 , $20,40,60,80,100,500,1000$ e $15000 \mathrm{hPa}$, para obtenção da $\theta$ CRAS. Foi calculada a diferença relativa proposta por Vachaud et al., (1985), tilizando os valores de $\theta$ e $\theta$ CRAS. A diferença relativa é dada por:

$$
D_{i j}=\frac{\left(\operatorname{var}_{i j}-\overline{\operatorname{var}}\right)}{\overline{\operatorname{var}}}
$$

Em que;

$D_{i j}$ - Diferença relativa da posição i no momento j; $\operatorname{var}_{i j}$ - Variável da posição i no momento j;

var - Média da variável de todas as posições no espaço i no momento j.

Para cada posição i, foi calculada a média do desvio relativo $\left(D M_{\mathrm{i}}\right)$ considerando os diversos momentos j.

Se a posição i apresentar um valor de $D M_{\mathrm{i}}$ igual à zero, esta posição permite inferir o valor médio de todas as posições i do domínio experimental. Portanto, $D M$ i fornece um indício de exatidão ao adotar a posição i como referência no processo de inferência estatística. $\mathrm{O}$ desvio padrão dos desvios relativos a todos os momentos $\mathrm{j}$ da posição i foi calculada, de acordo com Gonçalves et al., (1999). O desvio padrão é dado por:

$\sigma_{i}=\left(\frac{\sum_{j=1}^{n}\left(D_{i j}-D M_{i}\right)^{2}}{n}\right)^{0,5}$
Em que;

$D_{i j}$ - Desvio padrão da posição i;

$D M_{\text {i }}$ - Média do desvio relativo da posição i considerando diversos momentos $\mathrm{j}$.

Quanto menor o desvio padrão da posição i, maior a confiança desta para estimar a média geral (GONÇALVES et al., 1999), indicando a precisão desta posição. As variáveis foram analisadas a estatística descritiva, e assumindo a estacionaridade intrínseca das variáveis, foi procedido a análise geoestatística, sendo construídos os semivariogramas para descrever a estrutura de dependência espacial. Os valores de semivariância foram estimados pelos métodos dos momentos de Matheron:

$\gamma(h)^{*}=\frac{1}{2 N(h)} \sum_{i=1}^{N(h)}[Z(s i)-Z(s i+h)]^{2}$

Em que:

$\gamma(h)^{*}$ - semivariância estimada;

$N(h)$ - número de pares de valores da variável Z, medidos em pontos separados pela distância h;

$Z(s i)$ _ valor da variável $Z$ na localização $s i$, no espaço.

O escalonamento dovariograma foi realizado de acordo com a metodologia de Gonçalves et al., (1999) com o objetivo de comparar os semivariogramas. A correlação espacial foi confirmada pelo semivariograma cruzado como pode ser observada pelo modelo matemático abaixo.

$$
\gamma(h)_{12}^{*}=\frac{1}{2 N(h)} \sum_{i=1}^{N(h)}\left[Z_{1}(s i)-Z_{1}(s i+h)\right]\left[Z_{2}(s i)-Z_{2}(s i+h)\right]
$$

Em que;

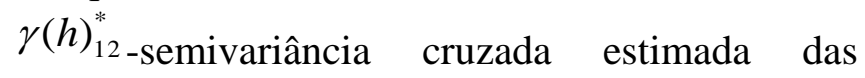
variáveis Z1 e Z2;

$N(h)$ - número de pares de valores da variável Z, medidos em pontos separados pela distância h;

$Z_{1}(s i)$ _ valor da variável $Z_{1}$ na localização $s i$, no espaço;
$Z_{2}(s i)$ - valor da variável ${ }^{Z_{2}}$ na localização $s i$, no espaço.

Com o mesmo objetivo de escalonar o semivariograma cruzado, foi utilizado o seguinte modelo matemático:

$\gamma(h)_{12}^{* * *}=\gamma(h)_{12}^{*}\left|\operatorname{cov}\left(Z_{1} ; Z_{2}\right)\right|^{-1}$ 
Em que;

$\gamma(h)_{12}^{*}$ - semivariância cruzada estimada das variáveis Z1 e Z2;

$\gamma(h)_{12}^{* *} \quad$ semivariância cruzada escalonada estimada das variáveis Z1 e Z2;

$\left|\operatorname{cov}\left(Z_{1} ; Z_{2}\right)\right|$ - módulo da covariância das variáveis $\mathrm{Z} 1$ e Z2.

A obtenção do semivariograma e semivariograma cruzado foram obtidos com o auxílio do software VARIOWIN (PANNATIER, 1996).

\section{RESULTADOS E DISCUSSÃO}


apresentadas a estatística descritiva dos dados experimentais dos experimentos A e B respectivamente. Pode-se observar que os dados das variáveis $\theta$ e $\theta$ CRAS de ambos os experimentos seguem uma distribuição normal a um nível de probabilidade de $5 \%$, confirmados pelo teste de KolmogorovSmirnov (K).

Tabela 1. Estatística descritiva dos diversos momentos avaliados para as variáveis $\theta$ e $\theta$ CRAS para o experimento A.

\begin{tabular}{cccccccc}
\hline Condição & Variável & Média & Mínimo & Máximo & CV \% & K & K crítico 5\% \\
\cline { 3 - 5 } L1 & & \multicolumn{3}{c}{$\mathrm{m}^{3} \mathrm{~m}^{3}$} & & & \\
L2 & $\theta$ & 0,35 & 0,30 & 0,38 & 5,72 & 0,10 & 0,19 \\
L3 & $\theta$ & 0,36 & 0,31 & 0,39 & 5,50 & 0,06 & 0,19 \\
L4 & $\theta$ & 0,35 & 0,29 & 0,39 & 6,72 & 0,08 & 0,19 \\
L5 & $\theta$ & 0,34 & 0,28 & 0,39 & 8,54 & 0,10 & 0,19 \\
L6 & $\theta$ & 0,32 & 0,25 & 0,38 & 9,47 & 0,13 & 0,20 \\
L7 & $\theta$ & 0,30 & 0,24 & 0,36 & 9,77 & 0,13 & 0,20 \\
L8 & $\theta$ & 0,29 & 0,23 & 0,36 & 10,24 & 0,10 & 0,19 \\
hPa0 & $\theta$ & 0,28 & 0,22 & 0,35 & 10,60 & 0,08 & 0,19 \\
hPa10 & $\theta$ CRAS & 0,44 & 0,38 & 0,52 & 7,58 & 0,10 & 0,19 \\
hPa20 & $\theta$ CRAS & 0,40 & 0,35 & 0,47 & 7,10 & 0,07 & 0,19 \\
hPa40 & $\theta$ CRAS & 0,39 & 0,33 & 0,45 & 7,42 & 0,06 & 0,19 \\
hPa100 & $\theta$ CRAS & 0,37 & 0,31 & 0,44 & 7,81 & 0,08 & 0,19 \\
hPa300 & $\theta$ CRAS & 0,35 & 0,30 & 0,43 & 8,76 & 0,09 & 0,19 \\
hPa800 & $\theta$ CRAS & 0,33 & 0,28 & 0,40 & 8,56 & 0,08 & 0,19 \\
hPa4000 & $\theta$ CRAS & 0,30 & 0,25 & 0,37 & 8,81 & 0,07 & 0,19 \\
hPa15000 & $\theta$ CRAS & 0,23 & 0,21 & 0,30 & 7,11 & 0,13 & 0,19
\end{tabular}

Tabela 2. Estatística descritiva dos diversos momentos avaliados para as variáveis $\theta$ e $\theta$ CRAS para o experimento B.

Condição Variável Média Mínimo Máximo $\mathrm{CV} \%$ K K crítico $5 \%$ 
CAMPO E NO LABORATÓRIO

\begin{tabular}{|c|c|c|c|c|c|c|c|}
\hline L1 & $\theta$ & 0,50 & 0,34 & 0,63 & 12,79 & 0,10 & 0,15 \\
\hline L2 & $\theta$ & 0,43 & 0,34 & 0,55 & 11,48 & 0,10 & 0,15 \\
\hline L3 & $\theta$ & 0,42 & 0,34 & 0,53 & 9,02 & 0,08 & 0,15 \\
\hline L4 & $\theta$ & 0,39 & 0,33 & 0,51 & 7,83 & 0,08 & 0,15 \\
\hline L5 & $\theta$ & 0,40 & 0,33 & 0,48 & 7,09 & 0,08 & 0,15 \\
\hline L6 & $\theta$ & 0,38 & 0,32 & 0,47 & 7,38 & 0,06 & 0,15 \\
\hline L7 & $\theta$ & 0,37 & 0,30 & 0,47 & 8,02 & 0,06 & 0,15 \\
\hline L8 & $\theta$ & 0,36 & 0,30 & 0,44 & 6,62 & 0,06 & 0,15 \\
\hline L9 & $\theta$ & 0,35 & 0,28 & 0,41 & 6,74 & 0,05 & 0,15 \\
\hline L10 & $\theta$ & 0,34 & 0,27 & 0,39 & 7,24 & 0,07 & 0,15 \\
\hline L11 & $\theta$ & 0,42 & 0,35 & 0,51 & 7,98 & 0,07 & 0,15 \\
\hline L12 & $\theta$ & 0,38 & 0,32 & 0,46 & 6,81 & 0,07 & 0,15 \\
\hline $\mathrm{hPa} 0$ & $\theta$ CRAS & 0,58 & 0,49 & 0,76 & 9,00 & 0,16 & 0,15 \\
\hline $\mathrm{hPa} 10$ & $\theta$ CRAS & 0,52 & 0,47 & 0,62 & 6,03 & 0,08 & 0,15 \\
\hline $\mathrm{hPa} 20$ & $\theta$ CRAS & 0,51 & 0,46 & 0,60 & 5,46 & 0,08 & 0,15 \\
\hline $\mathrm{hPa} 40$ & $\theta$ CRAS & 0,48 & 0,45 & 0,56 & 4,18 & 0,08 & 0,15 \\
\hline hPa60 & $\theta$ CRAS & 0,47 & 0,44 & 0,54 & 3,79 & 0,10 & 0,15 \\
\hline $\mathrm{hPa} 80$ & $\theta$ CRAS & 0,46 & 0,43 & 0,54 & 3,61 & 0,11 & 0,15 \\
\hline hPa100 & $\theta$ CRAS & 0,46 & 0,43 & 0,53 & 3,58 & 0,12 & 0,15 \\
\hline hPa500 & $\theta$ CRAS & 0,41 & 0,29 & 0,49 & 5,80 & 0,14 & 0,15 \\
\hline hPa8000 & $\theta$ CRAS & 0,34 & 0,32 & 0,41 & 4,18 & 0,12 & 0,15 \\
\hline hPa15000 & $\theta$ CRAS & 0,33 & 0,31 & 0,38 & 3,83 & 0,14 & 0,15 \\
\hline
\end{tabular}

Os valores médios de $\theta$, ao longo do processo de secamento do solo, mostram que ambos os experimentos estão dentro da faixa de valores de tensão de água no solo compatíveis para a umidade na capacidade de campo, considerando o valor $100 \mathrm{hPa}$ para o experimento A e $60 \mathrm{hPa}$ para o experimento $\mathrm{B}$, e para o limite do funcionamento de tensiômetros $(800 \mathrm{hPa})$. Tais condições garantem que o domínio de valores de $\theta$, relativos a uma amplitude de valores de tensão de água no solo, foram semelhantes para os dois experimentos. $\mathrm{Na}$ Tabela 3 são apresentadas as estatísticas descritivas das variáveis DM- $\theta$ e DM- $\theta$ CRAS para o experimento A e B. Para ambos os experimentos é possível adotar uma posição no espaço que represente a condição média do domínio, como pode ser confirmado pelo valor médio próximo à zero das variáveis DM- $\theta$ e DM- $\theta$ CRAS dos experimentos A e B.

Tabela 3. Estatística descritiva dos diversos momentos avaliados para as variáveis DM- $\theta$ e DM- $\theta$ CRAS para o experimento A e B.

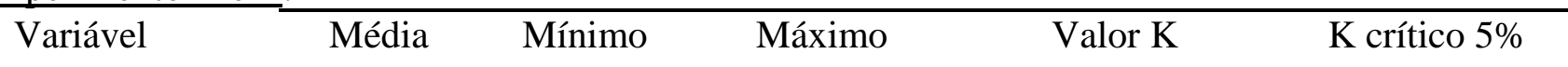




\begin{tabular}{|c|c|c|c|}
\hline DN & $-0,06$ & $-14,84$ & 15,69 \\
\hline
\end{tabular}
DM- $\theta$ CRAS
0,00
$-12,91$
22,08
0,08
0,19

Experimento B

$\begin{array}{cccccc}\text { DM- } \theta & 0,00 & -13,67 & 21,06 & 0,08 & 0,15 \\ \text { DM- } \theta \text { CRAS } & 0,00 & -6,36 & 16,31 & 0,19 & 0,15\end{array}$

A diferença relativa média e desvio padrão de $\theta$ e $\theta$ CRAS em função da posição (si) dos experimentos A e B são apresentados nas Figuras 1 e 2. Ao observar os valores de desvio padrão associado às variáveis $\mathrm{DM}-\theta$, os baixos valores desta variável para as duas condições experimentais sinalizam que a perda de água no solo apresenta uma taxa semelhante, com exceção de determinadas posições si, em todo o período do processo de secagem do solo. Essa constatação

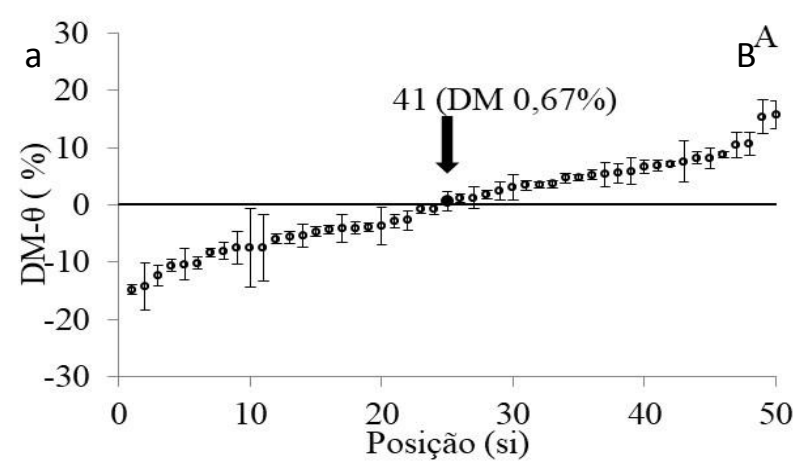

pode ser feita também para a variável DM- $\theta$ CRAS. Esses resultados evidenciam que a perda de água no solo está ligada às características inerentes da posição si em reter água no solo, não havendo interações significativas do mecanismo de retenção de água no solo com um provável movimento de água do solo do sentido horizontal adjacente ao ponto em questão que é realizada o monitoramento da umidade do solo.

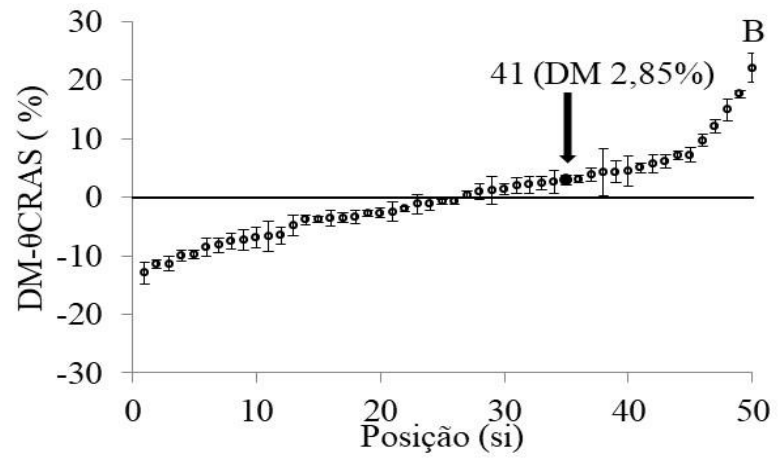

Figura 1. Diferença relativa média e desvio padrão de $\theta$ em função da posição (si) (A) e a diferença relativa média e desvio padrão da $\theta$ CRAS em função da posição (si) (B) do experimento A.
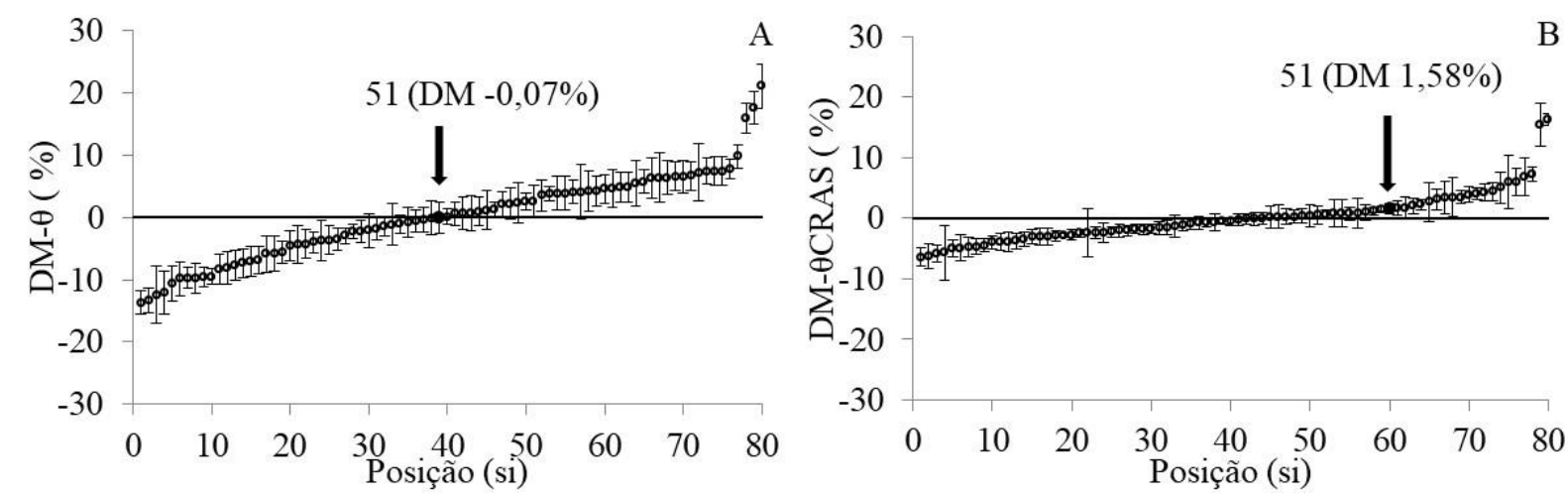

Figura 2. Diferença relativa média e desvio padrão de $\theta$ em função da posição (si) (A) e diferença relativa média e desvio padrão de $\theta$ CRAS em função da posição (si) (B) do experimento $B$.

A posição que apresentou o valor de DM- $\theta$ próximo à zero para os experimentos $\mathrm{A}$ e $\mathrm{B}$ foram 41 e 51 respectivamente, com valores de $0,67 \%$ para o experimento A e $-0,07 \%$ para o experimento B. Resultados semelhantes são apresentados por
Ávila et al., (2011). Verifica-se que ao realizar o monitoramento de água no solo considerando uma única posição no espaço, o erro associado à diminuição de esforço amostral deste processo é inferior a $1 \%$ considerando os dois experimentos. 
Contudo, ao adotar uma posição aleatória para realizar o monitoramento da umidade do solo, é possível cometer erros, que superestimam em $15,69 \%$ e $21,06 \%$ para os experimentos A e B respectivamente como podem ser observados na tabela 3.

Considerando as posições 41 do experimento A e 51 do experimento $\mathrm{B}$, ao observarmos a Figura 1 e 2, observa-se que o DM$\theta$ CRAS associado as respectivas posições, é de $2,85 \%$ e $1,58 \%$ do experimento A e B respectivamente. Portanto, verifica-se a viabilidade de aplicar a técnica da estabilidade temporal no estudo de $\theta$, identificando a posição si com o menor DM e posteriormente utilizar amostras desta posição para se determinar a CRAS, com erros inferiores a $3 \%$.

$\mathrm{Na}$ Figura 3 são apresentados os semivariogramas experimentais das variáveis $\theta$, $\theta$ CRAS, DM- $\theta$ e DM- $\theta$ CRAS do experimento A e $\theta$ e DM- $\theta$ do experimento B. No experimento A todas as variáveis abordadas apresentam estrutura de dependência espacial, com estruturas semelhantes entre si, sendo esta responsável por $60 \%$ da variação total. A semelhança da estrutura espacial das variáveis $\theta$ e DM- $\theta$ nos dois experimentos comprovam que a variável DM- $\theta$ foi influenciada pela estrutura espacial da variável $\theta$, devido a persistência dessa estrutura ao longo das diversas leituras realizadas. A persistência temporal da estrutura espacial da $\theta$ ao longo de um processo de secamento do solo pode ser corroborado em diversos trabalhos como Alves et al., (2011); Zhang et al., (2011); Cerqueira et al., (2014); Guimarães et al., (2010); Korres et al., (2015).
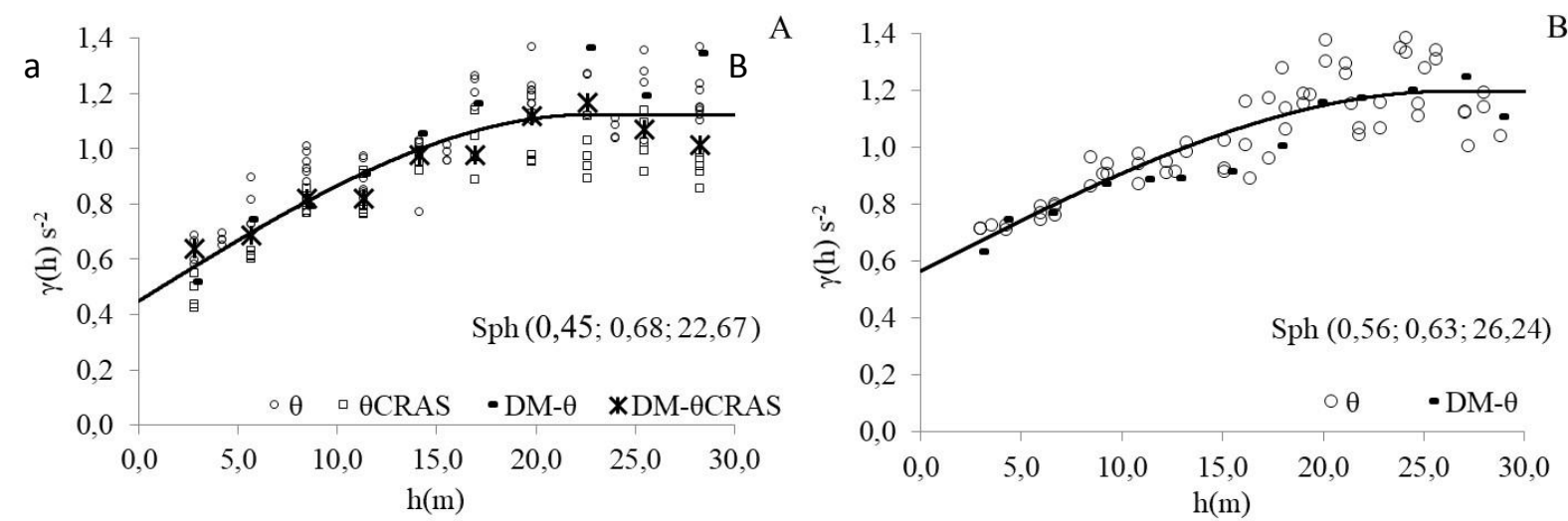

Figura 3. Semivariogramas escalonados das variáveis $\theta, \theta$ CRAS, DM- $\theta$ e DM- $\theta$ CRAS do experimento A (A), das variáveis $\theta$ e DM- $\theta$ do experimento $B(B)$.

No experimento B, somente foi encontrado estrutura de dependência espacial para as variáveis $\theta$ e DM- $\theta$, sendo ambas variáveis com uma estrutura de dependência responsável que explica $63 \%$ da variação total. A presença de estrutura de dependência espacial de tais variáveis confirma que o processo de retenção de água no solo no campo nos dois experimentos é distribuído de modo estocástico e estruturado no espaço.

De acordo com a Figura 4, por meio dos semivariogramas cruzados são confirmados geoestatisticamente a correlação espacial entre $\theta \mathrm{e}$ $\theta$ CRAS no experimento A e DM- $\theta$ e DM- $\theta$ CRAS no experimento $B$. Uma vez que a CRAS relaciona a energia de ligação da água no solo com a umidade do solo, podemos concluir por meio da correlação espacial realizada, que o processo de retenção de água no campo é devida a variabilidade de cargas elétricas existentes em cada área experimental, que interferem nesta relação (LAMBOOY, 1984; BOTHA; EISENBERG, 1993). Esses resultados justificam a estreita relação de valores de $\theta$ com $\theta$ CRAS para os dois experimentos, viabilizando o processo de inferência experimental 

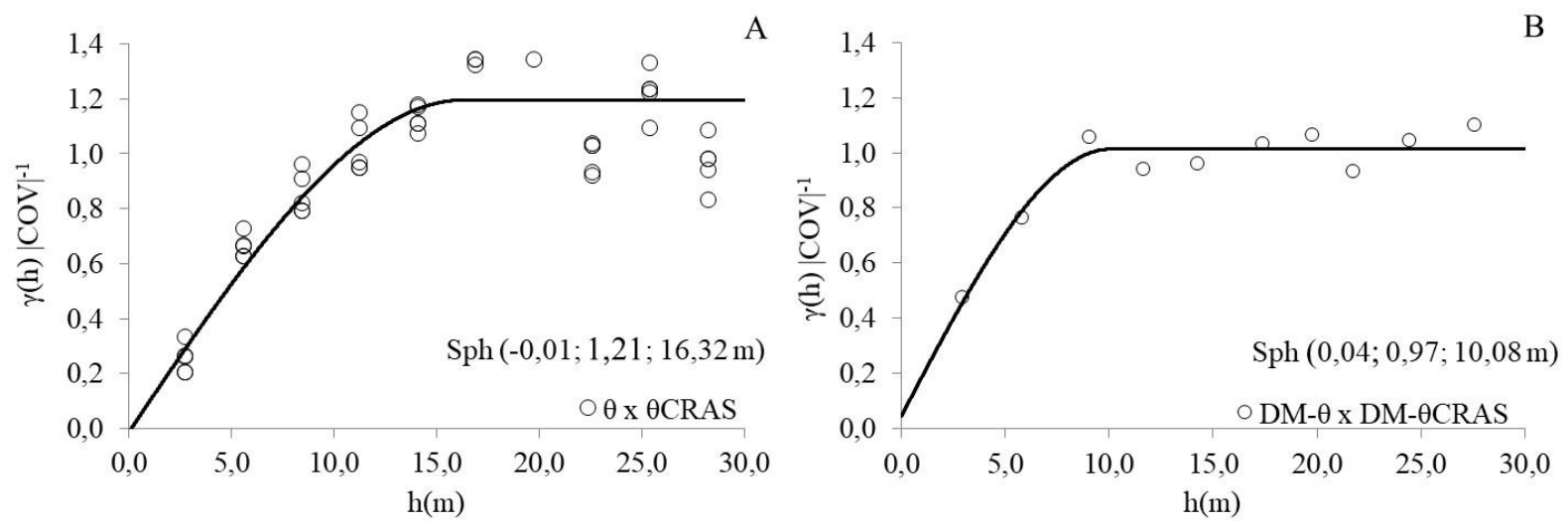

Figura 4. Semivariogramas cruzados escalonados do experimento A (A) e do experimento B (B).

\section{CONCLUSÕES}

As variáveis $\theta$ e $\theta$ CRAS apresentam estabilidade temporal e podem ser adotadas uma posição no espaço para o monitoramento da umidade do solo e uma CRAS que represente uma condição média da área experimental.

A variável $\theta$ pode ser utilizada para definir a posição da área experimental para coleta de amostras indeformadas de solo para a obtenção de uma curva de retenção de água no solo que represente uma condição média da área experimental.

\section{REFERÊNCIAS BIBLIOGRÁFICAS}

ALVES, W. W. A.; AZEVEDO, C. A. V.; NETO, J. D.; MATOS, J. A.; SILVA, S. S. Análise geoestatística da distribuição de água no solo, aplicada por sistema de irrigação por microaspersão. Revista Caatinga, v. 24, n. 2, p. 143-151, 2011.

ÁVILA, L. F.; MELLO, C. R.; MELLO J. M.; SILVA, A. M. Padrão espaço-temporal da umidade volumétrica do solo em uma bacia hidrográfica com predominância de Latossolos. Revista Brasileira de Ciência do Solo, v.35, p. 1801-1810, 2011. http://dx.doi.org/10.1590/S0100-06832011000500034.

BOTHA, A. D. P.; EISENBERG, B. E. Estimation of soil water retention from clay content and cation exchange capacity values of soils. South African Journal of Plant and Soil, v. 10, n. 3, p. 141-143, $1993 . \quad$ https:// doi.org/10.1080/02571862.1993.10634659.
CERQUEIRA, E. S. A.; QUEIROZ, D. M. de.; SANTOS, N. T.; CERQUIRA, N. M. M.; FILHO, R. R. G.; SANTOS, E. V. S. Spatial and temporal distribution of the water content of a Red-yellow argissol cultivated with beans (Phaseolus vulgaris L.) irrigated by center pivot. Revista Brasileira de Agricultura Irrigada, v. 8, n. 2, p. 188-198, 2014. https://doi.org/10.7127/rbai.v8n200247.

GONÇALVES, A. C. A.; FOLEGATTI, M. V; SILVA, A P. Estabilidade temporal da distribuição espacial da umidade do solo em área irrigada por pivô central. Revista Brasileira de Ciência do Solo, v. 23, n. 1, p. 155-164, 1999. http://dx.doi.org/10.1590/S0100-06831999000100019.

GONÇALVES, A. C. A.; TRINTINALHA, M. A.; FOLEGATTI, M. V.; REZENDE, R.; TORMENA, C. A. Spatial variability and temporal stability of water storage in a cultivated tropical soil. Bragantia, v. 69, p. 153-162, 2010. http://dx.doi.org/10.1590/S0006-87052010000500016.

GUIMARÃES, R. M. L.; GONÇALVES, A. C. A.; TORMENA, C. A; FOLEGATTI, M. V.; BLAINSKI, E. Variabilidade espacial de propriedades físico-hídricas de um Nitossolo sob a cultura do feijoeiro irrigado. Engenharia Agrícola, v.30, n.4, p. 657-669. 2010. http://dx.doi.org/10.1590/S010069162010000400010

KORRES, W.; TEICHENAU, T. G.; FEINER, P.; KOYAMA, C. N.; BOGENA, H. R.; CORNELISEN, T.; BAATZ, R.; HERBST, M.; DIEKKRUGER, B.; VEREECKEN, H.; SCHENEIDER, K. Spatio-temporal soil moisture patterns- A meta-analysis using plot to catchment 
scale data. Journal of hydrology, v. 520, p. 326341, 2015. https://doi.org/10.1016/j.jhydrol.2014.11.042.

LAMBOOY, A. M. Relationship between cation exchange capacity, clay content and water retention of Highveld soils. South African Journal of Plant and Soil, v. 1, n. 2, p. 33-38, 1984. https://doi.org/10.1080/02571862.1984.10634106.

MOHANTY, B. P.; MOUSLI, Z. Saturated hydraulic conductivity and soil water retention properties across a soil-slope transition. Water Resources Research, v. 36, n. 11, p. 3311, 2000. https://doi.org/10.1029/2000WR900216

PANNATIER, Y. VarioWin: Software for Spatial Data Analysis in 2D. New York: Springer, Verlag, 1996.

REZENDE, R.; GONÇALVES, A. C. A.; FREITAS, P. S. L. DE; FRIZZONE, J. A.; TORMENA, C. A.; BERTONHA, A. Influência da aplicação de água na uniformidade da umidade no perfil do solo. Acta Scientiarum - Agronomy, v. 24, n. 1978, p. 1553-1559, 2002. https://doi.org/10.4025/actasciagron.v24i0.2419.
SALVADOR, M. M. S.; LIBARDI, P. L.; BRITO, A. D. S.; MOREIRA, N. B. Estabilidade Temporal e Variabilidade espacial da distribuição da armazenagem de água no solo numa sucessão feijão/aveia-Preta. Revista Brasileira de Ciência do Solo, v. 36, p. 1434-1447, 2012. http://dx.doi.org/10.1590/S0100-06832012000500007.

VACHAUD G.; PASSERAT DE SILANS, A.; BALABANIS, P. \& VAUCLIN, M. Temporal stability of spatially measured soil water probability density function. Soil Science Society America Journal., 49:822-827, 1985. http:/l dx.doi.org/10.2136/sssaj1985.03615995004900040006x .

ZHANG, J. G.; CHEN, H. S.; SU, $\quad$ Y. $\quad$ R.; $\quad$ KONG. X. L.; ZHANG, W.; SHI, Y.; LIANG, H. B.; SHEN, G. M. Spatial variability and patterns of surface soil moisture in a field plot of karst area in southwest China. Plant Soil Environment, v. 57, n. 9, p. $409-417$, 2011. http://dx.doi.org/10.2136/sssaj1985.03615995004900040006x . 